Canad. Math. Bull. Vol. 44 (1), 2001 pp. 27-35

\title{
Normal Subloops in the Integral Loop Ring of an RA Loop
}

\author{
Edgar G. Goodaire and César Polcino Milies
}

\begin{abstract}
We show that an RA loop has a torsion-free normal complement in the loop of normalized units of its integral loop ring. We also investigate whether an RA loop can be normal in its unit loop. Over fields, this can never happen.
\end{abstract}

\section{Introduction}

Suppose $L$ is a loop (perhaps a group) which has an alternative loop ring over the ring $Z$ of rational integers. The loop of units (invertible elements) in $Z L$ is a Moufang loop which contains $L$ and it is of interest to see how $L$ sits inside $\mathcal{U}(Z L)$. If there exists a normal subloop $\mathcal{N}$ of $\mathcal{U}(Z L)$ such that $L \cap \mathcal{N}=\{1\}$ and $\mathcal{U}(Z L)= \pm L \mathcal{N}$, then $\mathcal{N}$ is called a normal complement of $L$. The search for a normal complement which is torsion-free is of great interest in group rings since the existence of such a subgroup implies a positive solution to the isomorphism problem: $Z G \cong Z H$ implies $G \cong$ H. (See Theorem 2.4 in this paper.) On the other hand, the two problems are not equivalent since, for example, the answer is "true" to the isomorphism problem for metabelian groups [Whi68] but "not true" to the existence of a torsion-free normal complement [RS83].

In Section 2 of this paper, we show that in the alternative loop ring of a Moufang loop $L$ which is not associative, $L$ indeed has a torsion-free normal complement. This has long been suspected and indeed has been established in the case that $L / L^{\prime}$ has exponent 2, 3, 4 or 6 [JL93], [GJM96, Proposition XII.4.1]. (While [GJM96] says "exponent at most 4", the result is true for exponent 6 as well since the proof requires only that the units of $Z\left[L / L^{\prime}\right]$ be trivial.)

In Section 3, we investigate a related question. Can $L$ ever be a direct factor of its unit loop? The answer is "never" for loop algebras over fields and "almost never" over the integers. See Theorems 3.4 and 3.1 respectively.

We now sketch briefly those facts about Moufang loops, alternative rings and loop rings which are required in this paper. Virtually all proofs can be found in the monograph [GJM96], but we endeavour also to cite original sources as much as possible.

An alternative ring is a ring which satisfies the identities $(y x) x=y x^{2}$ and $(x y) y=$ $x y^{2}$ and a Moufang loop is a loop which satisfies the identity

$$
(x y \cdot z) y=x(y \cdot z y) .
$$

Received by the editors April 30, 1999; revised February 10, 2000.

This research was supported by the Natural Sciences and Engineering Research Council of Canada, Grant No. OGP0009087, and by FAPESP and CNPq of Brasil, Proc. 1998/13127-7 and Proc. 300243/79$\mathrm{O}(\mathrm{RN})$ respectively.

AMS subject classification: Primary 20N05; Secondary 17D05, 16S34, $16 \mathrm{U} 60$.

(C)Canadian Mathematical Society 2001. 
Any group is a Moufang loop and any associative ring is alternative. The converses are "nearly" true. Alternative rings and Moufang loops are diassociative: the subring (or subloop) generated by any pair of elements is associative. In fact, if three elements in an alternative ring (or Moufang loop) associate, then they generate an associative ring (or a group).

If $L$ is a loop and $R$ is a commutative, associative ring with 1 , the loop ring $R L$ is defined just as in the case that $L$ is a group. If $R L$ is an alternative ring, then $L$ is necessarily Moufang, but the converse is not true in general. By definition, an RA loop is a Moufang loop whose loop rings are alternative, but not associative, over any ring $R$ [GJM96, Corollary IV.1.2]. The basic properties of RA loops are described in Section II.5.2 and Chapter IV of [GJM96]. See also [CG86] and [GP87].

Let $G$ be a nonabelian group with commutator subgroup $G^{\prime}=\{1, s\}$ of order 2, centre $\mathcal{Z}(G)$ and $G / \mathcal{Z}(G) \cong C_{2} \times C_{2}$, the Klein 4-group. Defining

$$
g^{*}= \begin{cases}g & g \in \mathcal{Z}(G) \\ s g & g \notin \mathcal{Z}(G)\end{cases}
$$

one can show that $g \mapsto g^{*}$ is an involution of $G$ (that is, an antiautomorphism of order 2). Let $L=G \cup G u$ for some indeterminate $u$ and extend the product in $G$ to $L$ by the rules

$$
\begin{gathered}
g(h u)=(h g) u \\
(g u) h=\left(g h^{*}\right) u \\
(g u)(h u)=g_{0} h^{*} g
\end{gathered}
$$

where $u^{2}=g_{0} \in \mathcal{Z}(G)$. Then $L$ is an RA loop which we denote $M\left(G, *, g_{0}\right)$. Of fundamental importance is the fact that a loop is RA if and only if it is a loop of the form $M\left(G, *, g_{0}\right)$ [GJM96, Theorem IV.3.1, Theorem III.3.3, Proposition III.3.6]. See also [CG86] and the introduction to [GP87]. We also note that if $L=M\left(G, *, g_{0}\right)$ is RA, then the centres $Z(L)$ and $Z(G)$ of $L$ and $G$, respectively, coincide and $\ell^{2} \in$ $Z(L)$ for all $\ell \in L$. Moreover, the unique nonidentity commutator of $G$ is a unique nonidentity commutator and a unique nonidentity associator of $L$.

A torsion element in a loop is an element of finite order. In an RA loop, the set of torsion elements forms a locally finite normal subloop which is actually finite if $L$ is finitely generated [GJM96, Lemma VIII.4.1], [GM95, Lemma 2.1]. A loop is torsion if every element is a torsion element and torsion-free if it has no nonidentity elements of finite order.

The set of units (invertible elements) of an alternative ring with 1 is a Moufang loop. In an integral loop ring $Z L$, the elements $\pm \ell, \ell \in L$, are clearly units. These are known as trivial units. A classical theorem of Higman says that if $G$ is a torsion group, the units of $Z G$ are all trivial if and only if $G$ is an abelian group of exponent 1, 2, 3, 4 or 6, or a hamiltonian 2-group [Hig40]. This theorem can be generalized to torsion loops $L$ for which $Z L$ is an alternative ring [GJM96, Theorem VIII.3.2], [GP86].

Of significance in this paper is the alternative vector matrix algebra $3(F)$ of Max Zorn. Here $F$ is a field and the elements of $3(F)$ are $2 \times 2$ matrices of the form $\left[\begin{array}{ll}a & x \\ y & b\end{array}\right]$, 
$a, b \in F, \mathrm{x}, \mathrm{y} \in F^{3}$. Such matrices are added in the obvious way, but multiplied according to the following variation of the usual rule,

$$
\left[\begin{array}{ll}
a_{1} & \mathrm{x}_{1} \\
\mathrm{y}_{1} & b_{1}
\end{array}\right]\left[\begin{array}{ll}
a_{2} & \mathrm{x}_{2} \\
\mathrm{y}_{2} & b_{2}
\end{array}\right]=\left[\begin{array}{cc}
a_{1} a_{2}+\mathrm{x}_{1} \cdot \mathrm{y}_{2} & a_{1} \mathrm{x}_{2}+b_{2} \mathrm{x}_{1}-\mathrm{y}_{1} \times \mathrm{y}_{2} \\
a_{2} \mathrm{y}_{1}+b_{1} \mathrm{y}_{2}+\mathrm{x}_{1} \times \mathrm{x}_{2} & b_{1} b_{2}+\mathrm{y}_{1} \cdot \mathrm{x}_{2}
\end{array}\right]
$$

where and $\times$ denote the dot and cross products respectively in $F^{3}$.

Zorn's algebra comes with a determinant function, $\left[\begin{array}{ll}a & \mathrm{x} \\ y & b\end{array}\right] \mapsto a b-\mathrm{x} \cdot \mathrm{y}$, and the units of $3(F)$ are precisely those matrices whose determinant is not zero. These units form a loop which is denoted $\operatorname{GLL}(2, F)$ and called the general linear loop. It was first studied by Paige [Pai56] who showed that the centre is $\pm\left[\begin{array}{ll}1 & 0 \\ 0 & 1\end{array}\right]$ and that, modulo its centre, $\operatorname{GLL}(2, F)$ is simple (and not associative).

Let $A$ denote a quaternion algebra over a field $F$ of characteristic different from 2. Let $\alpha$ be a nonzero element of $F$ and $u$ an element not in $A$. Let $\mathcal{C}=A+A u$ with obvious addition, but multiplication defined by the rule

$$
(a+b u)(c+d u)=\left(a c+\alpha d^{*} b\right)+\left(d a+b c^{*}\right) u
$$

where $a, b, c, d \in A$ and $q \mapsto q^{*}$ denotes any involution in $A$. The algebra $\mathcal{C}$ is called a Cayley-Dickson algebra. Such an algebra is alternative, but not associative; moreover, it is known that every such algebra is either a division algebra or isomorphic to Zorn's vector matrix algebra over F [GJM96, Corollary I.4.17], [ZSSS82, Theorem 2.4.7].

\section{Main Results}

Let $R$ be a commutative and associative ring with 1 . Let $A$ be a normal subloop of an RA loop (or a group) $L$ and let $\epsilon_{A}: R L \rightarrow R[L / A]$ denote the linear extension to $R L$ of the natural homomorphism $L \rightarrow L / A$. This map is a ring homomorphism whose kernel is the ideal

$$
\Delta(L, A)=\left\{\sum_{a \in A} \alpha_{a}(a-1) \mid \alpha_{a} \in R L\right\}
$$

Since $\epsilon_{A}$ is surjective, we have $R[L / A] \cong R L / \Delta(L, A)$. In the special case $A=L$, we write $\epsilon=\epsilon_{L}$, calling this the augmentation map on $R L$ and the element $\epsilon(\alpha)$ the augmentation of $\alpha \in R L$. Note that $\epsilon(\alpha)=\sum \alpha_{\ell}$ for $\alpha=\sum \alpha_{\ell} \ell \in R L$. The ideal $\Delta(L)=\Delta(L, L)$ is called the augmentation ideal of $L$. The identity $\ell_{1}\left(\ell_{2}-1\right)=$ $\left(\ell_{1} \ell_{2}-1\right)-\left(\ell_{1}-1\right)$ shows that

$$
\Delta(L)=\left\{\sum_{\ell \in L} \alpha_{\ell}(\ell-1), \alpha_{\ell} \in R\right\} .
$$

If $A$ is a normal subloop of $L, \Delta(L, A)=(R L) \Delta(A)$. We refer the reader to Section VI.1 of [GJM96] where the ideas of this paragraph are explained in more detail.

In the remainder of this section, we take $R=Z$, the ring of rational integers.

Lemma 2.1 Let A be a normal subloop of an RA loop $L$ and let $\delta \in \Delta(L, A)$. Then there exists $a \in A$ such that $\delta \equiv a-1(\bmod \Delta(L) \Delta(A))$. 
Proof The identity

$$
a b-1=(a-1)(b-1)+(a-1)+(b-1)
$$

and its consequence

$$
a^{-1}-1=-(a-1)-(a-1)\left(a^{-1}-1\right)
$$

imply that $a^{n}-1 \equiv n(a-1)\left(\bmod \Delta(A)^{2}\right)$ for any $a \in A$ and $n \in Z$. Thus, for any integers $n_{1}, n_{2}, \ldots, n_{k}$, and any $a_{1}, a_{2}, \ldots, a_{k} \in A$,

$$
\sum_{i=1}^{k} n_{i}\left(a_{i}-1\right) \equiv\left(\prod_{i=1}^{k} a_{i}^{n_{i}}\right)-1\left(\bmod \Delta(A)^{2}\right) .
$$

Let $\delta \in \Delta(L, A)$ and write $\delta=\sum_{i, j} \delta_{i j} \ell_{i}\left(a_{j}-1\right), \delta_{i j} \in Z$ Z, $\ell_{i} \in L, a_{j} \in A$. Since

$$
\ell(a-1)=(\ell-1)(a-1)+(a-1) \equiv a-1(\bmod \Delta(L) \Delta(A)),
$$

we have $\delta \equiv \sum_{j} \delta_{j}\left(a_{j}-1\right)(\bmod \Delta(L) \Delta(A)), \delta_{j}=\sum_{i} \delta_{i j}$. So $\delta \equiv a-$ $1(\bmod \Delta(L) \Delta(A)), a=\prod a_{j}^{\delta_{j}}$, by $(2.1)$.

Lemma 2.2 Let $L$ be an RA loop with centre $Z$. Then $L \cap(1+\Delta(L) \Delta(Z))=\{1\}$.

Proof We first remark that $L \cap(1+\Delta(L, Z))=Z$. On the one hand, for any $a \in Z$, $a=1+(a-1)$ with $a-1 \in \Delta(L, Z)$ implies that $z \subseteq 1+\Delta(L, Z)$. On the other hand, if $\ell \in 1+\Delta(L, Z)$, then $\ell-1 \in \Delta(L, Z)$ maps to 0 under the map $\epsilon_{Z}$. Since the image of $\ell-1$ is $\bar{\ell}-\overline{1}$, we have $\bar{\ell}=\overline{1}$, so $\ell \in Z$.

Next, we define an abelian group homomorphism $(\Delta(L, Z),+) \rightarrow Z$. To do so, we find a special way to represent the elements of $\Delta(L, Z)$. First write $L=\bigcup_{q \in Q} z q$ as the disjoint union of cosets of $Z$. Without loss of generality, we may assume that 1 is in the transversal $Q$. Any $\delta \in \Delta(L, Z)$ is an integral linear combination of terms of the form $\ell(a-1), \ell \in L, a \in Z$. Writing $\ell=q b$, with $q \in \mathcal{Q}$ and $b \in Z$, we have

$$
\ell(a-1)=q b(a-1)=q(b a-1)-q(b-1) .
$$

(In this calculation, we may associate freely since elements of $z$ associate with all other elements of $Z L$.) It follows that

$$
\delta=\sum_{i, j} \delta_{i j} q_{i}\left(a_{j}-1\right)
$$

$a_{j} \in Z, \delta_{i j} \in Z$ and $q_{i} \in Q$. Since $q_{i} a_{j}=q_{r} a_{s}$ implies $q_{i} Z=q_{r} Z$ and hence $q_{i}=q_{r}$, $a_{j}=a_{s}$, it is easy to see that the coefficients $\delta_{i j}$ in (2.2) are unique. Thus the map $\varphi: \Delta(L, Z) \rightarrow Z$ defined by

$$
\sum_{i, j} \delta_{i j} q_{i}\left(a_{j}-1\right) \mapsto \prod_{j} a_{j}^{\delta_{j}}, \quad \delta_{j}=\sum_{i} \delta_{i j}
$$


is well-defined and a homomorphism from the abelian group $(\Delta(L, Z),+)$ to $Z$. Under $\varphi$, the element

$$
(q b-1)(a-1)=q(b a-1)-q(b-1)-(a-1)
$$

maps to $(b a) b^{-1} a^{-1}=1$; thus $\Delta(L) \Delta(Z) \subseteq \operatorname{ker} \varphi$.

Now suppose $\ell \in 1+\Delta(L) \Delta(Z)$ and write $\ell=1+\delta, \delta \in \Delta(L) \Delta(Z)$. We have $\varphi(\delta)=1$. On the other hand, since $\Delta(L) \Delta(\mathcal{Z}) \subseteq \Delta(L, \mathcal{Z})$, the point made in the first paragraph gives $\ell \in \mathcal{Z}$ and so, by definition of $\varphi, \varphi(\delta)=\varphi(\ell-1)=\ell$. It follows that $\ell=1$.

Since the augmentation map $\epsilon: Z L \rightarrow Z$ is a homomorphism, if $\mu \in Z L$ is a unit, $\epsilon(\mu)= \pm 1$. We call $\mu$ normalized if $\epsilon(\mu)=+1$. The set $\mathcal{U}_{1}$ of normalized units in $Z L$ is a loop (containing $L$ ) and $\mathcal{U}(Z L)= \pm \mathcal{U}_{1}$. It follows that if $\mathcal{N}$ is a normal complement for $L$ in $\mathcal{U}_{1}$, then $\mathcal{N}$ is a normal complement for $L$ in the full unit loop.

In the proof of the theorem which follows, it is convenient to employ the term "support". If $\alpha=\sum_{\ell \in L} \alpha_{\ell} \ell, \alpha_{\ell} \in R$, is in a loop ring $R L$, the support of $\alpha$ is the set

$$
\operatorname{supp}(\alpha)=\left\{\ell \in L \mid \alpha_{\ell} \neq 0\right\}
$$

Theorem 2.3 Let L be a finite RA loop with centre Z. Then

$$
\mathcal{N}=(1+\Delta(L) \Delta(Z)) \cap \mathcal{U}_{1}(Z L)
$$

is a torsion-free normal complement for $L$ in the loop $\mathcal{U}_{1}$ of normalized units in $Z L$.

Proof The set $\Delta(L) \Delta(Z)$ is an ideal of $Z L$ because $\Delta(L)$ is an ideal and $\Delta(Z)$ is central. This implies that $\mathcal{N}$ is a normal subloop of $\mathcal{U}_{1}$. By Lemma 2.2, it remains only to prove that $L \mathcal{N}=\mathcal{U}_{1}$ and that $\mathcal{N}$ is torsion-free.

Let $\epsilon_{Z}: Z L \rightarrow Z[L / Z]$ denote the ring homomorphism which is the linear extension to $Z L$ of the natural loop homomorphism $L \rightarrow L / Z$ which maps $\ell$ to $Z \ell=\bar{\ell}$. Let $\bar{\alpha}$ denote the image in $Z[L / Z]$ of $\alpha \in Z L$ under the homomorphism $\epsilon_{z}$. Let $\mu \in \mathcal{U}_{1}$. Since $L / Z \cong C_{2} \times C_{2}$, the units in $Z[L / Z]$ are trivial by Higman's Theorem. Thus the image $\bar{\mu}$ of $\mu$ in $Z L / \Delta(L, Z) \cong Z[L / Z]$ is an element of the form $\pm \bar{\ell}, \ell \in L$, and, in fact, $+\bar{\ell}$ since the augmentation of $\bar{\mu}$ is +1 . So $\mu-\ell \in \operatorname{ker} \epsilon_{Z}=\Delta(L, Z)$ and we have $\mu=\ell(1+\delta)$ for some $\delta \in \Delta(L, Z)$. By Lemma $2.1, \delta \equiv a-1(\bmod \Delta(L) \Delta(Z))$ for some $a \in Z$, so, for some $\delta_{1}, \delta_{2} \in \Delta(L) \Delta(Z)$, we have $\mu=\ell\left(1+a-1+\delta_{1}\right)=$ $\ell\left(a+\delta_{1}\right)=\ell a\left(1+\delta_{2}\right) \in L \mathcal{N}$. Thus $L \mathcal{N}=\mathcal{U}_{1}$.

We now show that $\mathcal{N}$ is torsion-free. Because a torsion unit with a central element in its support is necessarily an element of $L$ [GJM96, Corollary VIII.1.2], [GM89, Corollary 2.2] and in view of Lemma 2.2, it suffices to show that each element of $1+\Delta(L) \Delta(Z)$ has a central element in its support. Let then $\alpha \in 1+\Delta(L) \Delta(Z)$. Using (2.3), we may write

$$
\alpha=1+\sum_{q \in \mathcal{Q}, a \in \mathcal{Z}} \alpha_{q a} q(a-1)+\sum_{b \in \mathcal{Z}} \beta_{b}(b-1),
$$


$\alpha_{q a}, \beta_{b} \in Z, a, b \in Z$ and $\mathcal{Q}$ a transversal of $Z$ in $L$ containing 1 . In the right hand side of (2.4), a fixed $a_{0} \in Z, a_{0} \neq 1$, has coefficient $\alpha_{1 a_{0}}+\beta_{a_{0}}$. Thus, if $\alpha_{1 a_{0}}+\beta_{a_{0}} \neq 0$, then $a_{0}$ is in the support of $\alpha$ and we have the desired result. On the other hand, if $\alpha_{1 a}+\beta_{a}=0$ for all $a \in Z$, then the coefficient of 1 on the right side of (2.4) is

$$
1-\sum_{a \in \mathcal{Z}} \alpha_{1 a}-\sum_{b \in \mathcal{Z}} \beta_{b}=1 \neq 0
$$

so 1 is in the support of $\alpha$.

The original proof of the following "isomorphism theorem" appeared in [GM89]. See also [GJM96, Theorem IX.1.1].

Theorem 2.4 Let $L$ and $L_{1}$ be finite RA loops and suppose that $Z L_{1} \cong Z L$. Then $L_{1} \cong L$.

Proof Note first that $L$ and $L_{1}$ have the same order, since each is the rank of the same free Z-module. Suppose $\varphi: Z L_{1} \rightarrow Z L$ is the given isomorphism and let $\mathcal{N}$ be a torsion-free normal complement for $L_{1}$ in $\mathcal{U}_{1}\left(Z L_{1}\right)$. Then $\varphi(\mathcal{N})$ is torsionfree in $\mathcal{U}_{1}(Z L)$ and so $L \cap \varphi(\mathcal{N})=\{1\}$. Since $\left[\mathcal{U}_{1}(Z L): \varphi(\mathcal{N})\right]=\left|L_{1}\right|=|L|=$ $[L \varphi(\mathcal{N}): \varphi(\mathcal{N})]$, we have $\mathcal{U}_{1}(Z L)=L \varphi(\mathcal{N})$. Thus

$$
L_{1} \cong \frac{\mathcal{U}_{1}\left(Z L_{1}\right)}{\mathcal{N}} \cong \frac{\mathcal{U}_{1}(Z L)}{\varphi(\mathcal{N})} \cong \frac{L \varphi(\mathcal{N})}{\varphi(\mathcal{N})} \cong \frac{L}{L \cap \varphi(\mathcal{N})}=L
$$

\section{Related Questions}

In view of Theorem 2.3, it is natural to ask if $L$ can ever be a direct factor of $\mathcal{U}_{1}$. As we shall see, with $L$ finite, this happens only when $\mathcal{U}_{1}=L$.

Theorem 3.1 Let $L$ be a finite RA loop. Then $L$ is normal in $\mathcal{U}(Z L)$ if and only if $\mathcal{U}(Z L)$ is itself an RA loop and this occurs if and only if $U(Z L)= \pm L$.

Proof If $\mathcal{U}(Z L)$ is an RA loop, then the torsion units form a subloop of $Z L$, so the loop $T$ of torsion elements in $L$ is either an abelian group or a hamiltonian Moufang 2-loop [GJM96, Corollary XII.2.14], [GM95, Theorem 3.1]. Here, $T=L$ is a hamiltonian Moufang 2-loop. In this case, the generalization of Higman's Theorem to alternative loop rings says $\mathcal{U}(Z L)= \pm L$, so $L$ is certainly normal in $\mathcal{U}(Z L)$.

Conversely, assume that $L$ is normal in its unit loop. If $\nu \in \mathcal{U}(Z L)$ and $\ell \in L$, then $\nu^{-1} \ell \nu$ is an element of the finite set $L$. It follows that each $\mu=\sum_{\ell \in L} \mu_{\ell} \ell \in \mathcal{U}(Z L)$ has just finitely many conjugates of the form $\nu^{-1} \mu \nu$. Such a loop is called FC and it is known that if $\mathcal{U}(Z L)$ is FC, then $\mathcal{U}(Z L)$ is RA [GJM96, Corollary XII.2.14], [GM95, Theorem 3.3].

The final part of the proof follows from the fact that if $L$ is finite, then $\mathcal{U}(Z L)$ is $R A$ if and only if $L$ is a hamiltonian Moufang 2-loop [GJM96, Corollary XII.2.14] and so $\mathcal{U}(\mathrm{ZL})= \pm L$ [GJM96, Theorem VIII.3.2] [GP86, Theorem 7]. 
Remark 3.2 The condition that $\mathcal{U}(Z L)$ be RA is equivalent to many other conditions on this unit loop, including nilpotency and the $n$-Engel and FC properties [GJM96, Corollary XII.2.14], [GM95, Theorem 3.3].

Example 3.3 There do indeed exist RA loops $L$ with $\mathcal{U}(Z L) \neq \pm L$ also RA. By Corollary XII.2.14 of [GJM96] (see also [GM95, Theorem 3.3]), it is sufficient to construct an RA loop with a torsion subloop $T$ which is an abelian group with the property that if $x \in L$ does not centralize $T$, then $x^{-1} t x=t^{-1}$ for all $t \in T$. To construct such an $L$, let $A=\langle s\rangle \times\langle b\rangle, s^{2}=1$, be the direct product of a cyclic group of order 2 and an infinite cyclic group $\langle b\rangle$. Let $G$ be the group generated by $A$ and elements $x, y$ subject to the relations

$$
a x=x a, a y=y a \text { for } a \in A, \quad x^{2}=s, y^{2}=b,(x, y)=s .
$$

Then $G^{\prime}=\{1, s\}, Z=A$ and $G / Z=\langle x\rangle \times\langle y\rangle \cong C_{2} \times C_{2}$. The loop $M(G, *, b)=$ $G \cup G u$, where $u^{2}=b$, is RA with torsion subloop $T=\langle s, x\rangle$ and $y^{-1} x^{-1} y=$ $(y, x) x^{-1}=s x^{-1}=x$.

As we now show (Theorems 3.4 and 3.6), in contrast to Theorem 3.1, $L$ is never normal in the unit loop of a loop algebra over a field $F$ and $U(F L)$ is never RA.

Theorem 3.4 Let $L$ be a finite RA loop and let $F$ be a field. Then $L$ is not normal in U(FL).

Proof Assume that $L$ is normal in $\mathcal{U}(F L)$. As in the proof of Theorem 3.1, $\mathcal{U}(F L)$ must be an FC loop, so $F$ is finite [GJM96, Theorem XIII.3.5], [GM96a], say of characteristic $p>0$. Since $L$ is finite, we can write $L=L_{2} \times L_{2^{\prime}}$ as the direct product of an RA 2-loop $L_{2}$ and an abelian group $L_{2^{\prime}}$ [GJM96, Proposition V.1.1], [CG86, Theorem 6]. If $\ell \in L_{2}$ and $\mu \in \mathcal{U}(F L)$, then $\mu^{-1} \ell \mu$ is a 2-element of $L$ and hence in $L_{2}$. It follows that we may assume that $L$ is a 2-loop.

Let $F_{p}$ denote the field of $p$ elements and suppose first that $p \neq 2$. Then $F L$ is semisimple and hence the direct sum of fields $F_{i}$ and Cayley-Dickson algebras $A_{i}$ [GJM96, Corollary VI.4.8] [GM96b, Theorem 2.8], so U(FL) is the product of the unit loops of these $F_{i}$ and $A_{i}$. Since each $A_{i}$ is finite, it is not a division algebra. (A finite alternative division ring is commutative by Wedderburn's Theorem, since the subring generated by any two elements is a finite division ring, and hence associative [ZSSS82, Theorem 3, p. 143].) Thus each $A_{i}$ is a Zorn's vector matrix algebra over a field $K$ and the projection of $\pm L$ in $A_{i}$ is a subloop of the unit loop $U\left(A_{i}\right)$ of $A_{i}$. Since $L$ is normal in $\mathcal{U}(F L)$, the projection of $\pm L$ is normal in $U\left(A_{i}\right)$ which, as explained in the introduction, is the general linear loop $\operatorname{GLL}(2, K)$. Since $\pm\left[\begin{array}{ll}1 & 0 \\ 0 & 1\end{array}\right]$ is the only nontrivial normal subloop of $\operatorname{GLL}(2, K)$, either the image of $\pm L$ is $\operatorname{GLL}(2, K)$ or it is central (perhaps trivial). The first possibility cannot occur, however, since $G$ is a nonabelian normal subloop in $L$ and there are no such subloops of $\operatorname{GLL}(2, K)$. The second possibility cannot occur either since the image of $L$ in $U(F L)$ is not central. Thus $p=2$.

Let $F_{2}$ denote the field of 2 elements. Clearly $F_{2} L \subseteq F L$ and $L$ is normal in $U\left(F_{2} L\right)$. Let $\epsilon: F_{2} L \rightarrow F_{2}$ be the augmentation map. Since $\epsilon$ is a ring homomorphism, if $\alpha \epsilon$ 
$F_{2} L$ is a unit, necessarily $\epsilon(\alpha)=1$. On the other hand, if $\alpha \in F_{2} L$ has augmentation 0 , then $\alpha$ lies in the augmentation ideal $\Delta(L)$ which is known to be nilpotent [Goo95], [MZ, Theorem 3.4]. In particular, $\alpha$ is not a unit. Thus $\alpha \in F_{2} L$ is a unit if and only if $\epsilon(\alpha)=1$. Let $g, h \in L$ be two elements which do not commute. Then $\mu=1+g+h$ is a unit in $F_{2} L$ which does not commute with $g$, so $\mu^{-1} g \mu=t \in L$. We have $g \mu=\mu t$, implying $g+g^{2}+g h=t+g t+h t$. Now $g^{2} \neq g, g^{2} \neq g h$ and $g^{2} \neq g t$; thus $g^{2}=t$ or $g^{2}=h t$. If $g^{2}=t$, then $t$ is central (the square of any element of $L$ is central) and so is $g=\mu t \mu^{-1}=t$, a contradiction. So $g^{2}=h t$ which, after cancellation, gives $g+g h=t+g t$ and $g \in\{g h, t, g t\}$, which is not true.

Remark 3.5 Let $R$ be any commutative associative ring with 1 and of characteristic $p>0$. Since $R$ contains $F_{p}$, the proof of Theorem 3.4 shows that $L$ is never normal in $U(R L)$. Such is not the case with group rings over finite rings; for example, the symmetric group $S_{3}$ is normal in $U\left(F_{2} S_{3}\right)$ [Seh78, Section 6.2, p. 215].

Theorem 3.6 Let L be a finite RA loop and $F$ a field. Then $U(F L)$ is not RA.

Proof Suppose $\mathcal{U}(F L)$ is an RA loop. Since $L$ contains an RA 2-loop, we may assume that $L$ itself is a 2-loop.

Suppose that the characteristic of $F$ is different from 2. Again, $U(F L)$ is the product of the unit groups of fields and Cayley-Dickson algebras. Let $A$ be one of the CayleyDickson algebras and $\mathcal{U}=\mathcal{U}(A)$ its unit loop. As a subloop of the RA loop $U(F L), \mathcal{U}$ is clearly RA too. If $A$ has zero divisors, then $A$ is a Zorn's vector matrix algebra and $\mathcal{U}$ is a general linear loop. This loop is not RA for a variety of reasons; for instance, it contains the general linear group and hence does not possess a unique nonidentity commutator. On the other hand, if $A$ is a division algebra, then $\mathcal{U}=A \backslash\{0\}$ contains $Q_{8}$, the quaternion group of order 8. In $Q_{8}$, the unique nonidentity commutator is -1 , so this is the unique nonidentity commutator in $\mathcal{U}$. Since $i+j$ and $i$ do not commute, we would have $(i+j) i=-i(i+j)$, giving $2 i^{2}=0$, a contradiction. Thus $\operatorname{char} F=2$.

Let $F_{2}$ denote the field of 2 elements. Since $F_{2} L \subseteq F L, \mathcal{U}\left(F_{2} L\right)$ is an RA loop. In particular, this unit loop has a unique nonidentity commutator-associator which is necessarily the unique nonidentity commutator-associator, $s$, of $L$. As noted in the proof of Theorem 3.4, $\alpha \in F_{2} L$ is a unit if and only if $\epsilon(\alpha)=1$, where $\epsilon: F_{2} L \rightarrow F_{2}$ is the augmentation map. Let $g$ and $h$ be two elements of $L$ which do not commute. Thus $h g=s g h$. Then $1+g+h$ is a unit which does not commute with $g$, so the commutator of these two elements is also $s$. The equation $(1+g+h) g=s g(1+g+h)$ implies $g+g^{2}=s g+s g^{2}$ and hence $1+g=s+s g$, an impossibility since $g \neq 1, g \neq s$ and $g \neq s g$.

Acknowledgements The authors thank the referee for helping them to improve the original version of this paper. We also thank M. M. Parmenter for a comment which substantially shortened the proof of Lemma 2.2.

Added in proof S. O. Juriaans has recently informed us that Theorems 2.3 and 2.4 also appear in a paper of his with L. G. X. de Barros [dBJ97]. 


\section{References}

[CG86] Orin Chein and Edgar G. Goodaire, Loops whose loop rings are alternative. Comm. Algebra (2) 14(1986), 293-310.

[dBJ97] Luiz G. X. de Barros and Stanley O. Juriaans, Units in alternative integral loop rings. Resultate Math. 31(1997), 266-281.

[GJM96] E. G. Goodaire, E. Jespers, and C. Polcino Milies, Alternative loop rings, North-Holland Math. Studies 184, Elsevier, Amsterdam, 1996.

[GM89] Edgar G. Goodaire and César Polcino Milies, Torsion units in alternative loop rings. Proc. Amer. Math. Soc. 107(1989), 7-15.

[GM95] On the loop of units of an alternative loop ring. Nova J. Algebra Geom. (3) 3(1995), 199-208.

[GM96a] - Finite conjugacy in alternative loop algebras. Comm. Algebra (3) 24(1996), 881-889.

[GM96b] Finite subloops of units in an alternative loop ring. Proc. Amer. Math. Soc. (4) 124(1996), 995-1002.

[Goo95] Edgar G. Goodaire, The radical of a modular alternative loop algebra. Proc. Amer. Math. Soc. (11) 123(1995), 3289-3299.

[GP86] Edgar G. Goodaire and M. M. Parmenter, Units in alternative loop rings. Israel J. Math. (2) 53(1986), 209-216

[GP87] Edgar G. Goodaire and M. M. Parmenter, Semi-simplicity of alternative loop rings, Acta Math. Hungar. (3-4) 50(1987), 241-247.

[Hig40] Graham Higman, The units of group rings. Proc. London Math. Soc. (2) 46(1940), 231-248.

[JL93] Eric Jespers and Guilherme Leal, A characterization of the unit loop of the integral loop ring Z $\left[M_{16}(Q, 2)\right]$. J. Algebra (1) 155(1993), 95-109.

[MZ] C. Polcino Milies and Albertina Zatelli, Nilpotent elements and ideals in alternative loop rings. East West J. Math., to appear.

[Pai56] Lowell J. Paige, A class of simple Moufang loops. Proc. Amer. Math. Soc. 7(1956), 471-482.

[RS83] K. W. Roggenkamp and L. L. Scott, Units in metabelian group rings: non-splitting examples for normalized units. J. Pure Appl. Algebra 27(1983), 299-314.

[Seh78] S. K. Sehgal, Topics in group rings, Marcel Dekker, New York, 1978.

[Whi68] A. Whitcomb, The group ring problem, Ph.D. thesis, Chicago, 1968.

[ZSSS82] K. A. Zhevlakov, A. M. Slin'ko, I. P. Shestakov, and A. I. Shirshov, Rings that are nearly associative. Academic Press, New York, 1982, translated by Harry F. Smith.

Memorial University of Newfoundland St. John's, Newfoundland A1C $5 S 7$

e-mail: edgar@math.mun.ca
Instituto de Matemática e Estatística

Universidade de São Paulo

Caixa Postal 66.281

CEP 05315-970

São Paulo SP

Brasil

e-mail: polcino@ime.usp.br 